\title{
Recent Trends in RSV Immunoprophylaxis: Clinical Implications for the Infant
}

\author{
Santiago Acero-Bedoya, BS ${ }^{1}$ Phillip S. Wozniak, BS ${ }^{2}$ \\ Asuncion Mejias, MD, PhD, MSCS $1,4,5$ \\ ${ }^{1}$ Center for Vaccines and Immunity, The Research Institute at \\ Nationwide Children's Hospital, The Ohio State University College of \\ Medicine, Columbus, Ohio \\ ${ }^{2}$ Center for Perinatal Research, The Research Institute at Nationwide \\ Children's Hospital, The Ohio State University College of Medicine, \\ Columbus, Ohio \\ ${ }^{3}$ Division of Neonatology, Department of Pediatrics, Nationwide \\ Children's Hospital, The Ohio State University College of Medicine, \\ Columbus, Ohio \\ ${ }^{4}$ Division of Infectious Diseases, Nationwide Children's Hospital, \\ The Ohio State University College of Medicine, Columbus, Ohio \\ ${ }^{5}$ Departamento de Farmacologia y Pediatria, Facultad de Medicina de \\ Malaga, Universidad de Malaga (UMA), Spain
}

\author{
Pablo J. Sánchez, MD ${ }^{2,3,4}$ Octavio Ramilo, MD ${ }^{1,4}$
}

Am J Perinatol 2019;36(suppl S2):S63-S67.

\begin{abstract}
Address for correspondence Asuncion Mejias, MD, PhD, MSCS, Division of Pediatric Infectious Diseases and Center for Vaccines and Immunity, The Research Institute at Nationwide Children's Hospital and The Ohio State University College of Medicine, Columbus, $\mathrm{OH}$ 43205 (e-mail: Asuncion.Mejias@Nationwidechildrens.org).
\end{abstract}

\author{
Abstract \\ Keywords \\ - respiratory syncytial \\ virus \\ - palivizumab \\ - premature infants \\ - disease severity \\ - hospitalizations
}

Respiratory syncytial virus (RSV) remains the leading cause for hospitalizations in infants worldwide, resulting in significant health and financial burden. Since 1998, the humanized monoclonal antibody palivizumab remains the only available option licensed for the prevention of severe RSV disease in high-risk children, namely premature infants and those with chronic lung disease and congenital heart disease. In 2014, the American Academy of Pediatrics modified the recommendations on the use of RSV prophylaxis in these high-risk children, and limited its use to premature infants born at $<28$ weeks' gestational age (wGA). Following this last guidance update, studies have confirmed that premature infants of 29 to 34 WGA remain at high risk for severe RSV disease, especially those of younger chronologic age. New and more cost-effective strategies are being developed that would help alleviate both the health and financial burden associated with severe RSV disease.
Respiratory syncytial virus (RSV) accounts for over 30 million new cases of lower respiratory tract infections (LRTI) per year worldwide, resulting in 3.2 million hospital admissions and nearly 60,000 deaths in children $<5$ years of age. ${ }^{1}$ In the United States alone, it is estimated that 132 to 172,000 children below the age of 5 are hospitalized annually due to an RSV-related illness, representing the leading cause of hospitalization in infants. ${ }^{2}$ Epidemiologic studies have consistently shown that premature infants and children with chronic lung disease (CLD)/bronchopulmonary dysplasia or hemodynamically significant congenital heart disease (CHD) are at increased risk for developing severe RSV disease. ${ }^{3}$ The mechanisms for this increased morbidity are not well understood, and studies suggest that in addition to the immature or under-developed lungs, a dysregulated immune response may play a role. In fact, young infants with RSV infection compared with older infants ( $>6$ months), had lower expression of blood and mucosal interferon that was associated with longer hospital stay and duration of supplemental oxygen. ${ }^{4}$ Nevertheless, the high disease burden associated with RSV emphasizes the need for the development of safe and effective preventive and therapeutic interventions.

\section{Viral Targets for Monoclonal Antibodies}

RSV is an enveloped, negative-sense, single-stranded ribonucleic acid (RNA) virus that exists as two antigenic subtypes, A and B, which can cocirculate during the same season
Copyright $\odot 2019$ by Thieme Medical Publishers, Inc., 333 Seventh Avenue, New York, NY 10001, USA. Tel: +1(212) 584-4662.
DOI https://doi.org/ 10.1055/s-0039-1691803. ISSN $0735-1631$. 
and exhibit genome-wide sequence divergence. RSV RNA genome encodes 11 proteins, of which the fusion (F) and attachment $(G)$ glycoproteins are crucial for infectivity and viral pathogenesis as they carry the antigenic determinants that elicit neutralizing antibodies. Of these two, the F protein is the preferred target for vaccine, monoclonal antibodies $(\mathrm{mAb})$, and antiviral development because it plays a key role in host cell viral entry, is highly conserved within and among RSV A and B subtypes, and has six antigenic sites that elicit the production of high-potency neutralizing antibodies. In contrast, the G protein is not well conserved among RSV A and B strains, and is highly glycosylated, and thus it is only recognized by few neutralizing antibodies that are directed to its central domain. These facts have reduced the enthusiasm for considering the $G$ protein as a potential therapeutic target.

Our understanding of the fusion protein in its two conformations, prefusion (preF) and postfusion (postF), has revolutionized the field of RSV biology. ${ }^{5}$ PreF, the active form of the F protein on the virion, is metastable and once it enters in contact with the host cells switches unpredictably to the stable postF conformation. Once the postF protein is folded, it cannot return to the preF form. Antibodies that bind to preF are more efficient at neutralizing RSV than those against postF. As examples, antibodies against antigenic site $\emptyset$, a preF-specific epitope, are 100 times more potent in vitro than palivizumab-that binds to antigenic site II, present in both $\mathrm{F}$ conformations. On the other hand, antibodies against antigenic site I, exclusively present in postF, show weak or no neutralization. Thus, the two conformations of the F protein, preF and postF, have not only common but also specific antigenic sites that elicit the production of different potency neutralizing antibodies.

\section{History of RSV Immunoprophylaxis}

There are currently no licensed vaccines for the prevention or amelioration of RSV infection, and treatment remains symptomatic. In 1998, palivizumab, a humanized mAb directed against antigenic site II, present in the F glycoprotein in its preF and postF conformations, was licensed for the prevention of serious RSV LRTI in high-risk infants and children. ${ }^{6}$ In the original double-blind, placebo-controlled clinical trial, palivizumab prophylaxis decreased overall hospitalization rates by $55 \%$ in infants $\leq 35$ weeks' gestational age (wGA) and in children $<24$ months of age with CLD. In addition, infants in the prophylaxis arm had significantly shorter duration of both RSV-associated hospitalizations and of supplemental oxygen, lower disease severity scores, and less pediatric intensive care unit (PICU) admissions. ${ }^{4}$ Subsequently in 2003, Feltes et $\mathrm{al}^{7}$ conducted a similar randomized placebo-controlled international study that evaluated palivizumab prophylaxis in children $<24$ months of age with CHD. The study showed that RSV immunoprophylaxis resulted in a $45 \%$ reduction in all of RSV-associated hospitalizations in these children, as well as a significant reduction in the duration of RSV hospitalizations and days of supplemental oxygen in palivizumab recipients. A third randomized, placebo-controlled clinical trial recently was conducted in premature infants born at 33 to 35 wGA in the Netherlands. The study showed that in addition to a $61 \%$ decrease in the number of wheezing days during the first year of life (primary end point), RSV prophylaxis was associated with an $82 \%$ reduction in RSV-associated hospitalizations. ${ }^{8}$ Taken together, these and other observational studies have shown that the use of mAb against RSV in high-risk infants is associated with a significant reduction in the acute and possibly long-term morbidity associated with RSV.

Since its initial licensure, the American Academy of Pediatrics (AAP) has updated the RSV prophylaxis guidance on five occasions. In its latest iteration in 2014, the AAP Committee on Infectious Diseases, and mainly because of costs, limited RSV prophylaxis to premature infants born at $<29$ wGA, infants $<32$ wGA with CLD, and infants younger than 12 months with CHD (-Table 1). Following the implementation of the 2014 AAP guidance, several observational studies have been conducted to determine the impact of these changes in populations who previously were eligible for RSV immunoprophylaxis.

\section{Current Trends in RSV-Associated Morbidity in Premature Infants}

We recently conducted a single-center retrospective study to examine the frequency and clinical impact of RSV-associated hospitalizations in infants $<12$ months of age born at 29 to 34 wGA during the seasons before and after the implementation of the 2014 AAP guidance update (S1 [2013-2014], preguidance update; S2 [2014-15], postguidance update). ${ }^{9}$ We found that there was an increase in RSV hospitalizations and associated morbidity among 29 to 34 wGA infants of younger chronological age following implementation of the 2014 palivizumab prophylaxis guidance. Specifically, of 1,063 RSV hospitalizations in infants $<12$ months old, 7.1\% (34/482) in S1 and 9.8\% (57/581) in S2 occurred in 29 to 34 wGA infants. On the other hand, 29 to $34 \mathrm{wGA}$ infants who were $<6$ months old constituted 3.5\% of RSV hospitalizations in $\mathrm{S} 1$ versus $7.1 \%$ in S2 $(p=0.01)$. In addition, among these premature infants who were $<3$ months old, supplemental oxygen administration (40 vs. $79 \%$ ), PICU admission (30 vs. $68 \%$ ), mechanical ventilation (10 vs. $53 \%$ ), duration of hospitalization (1.8 vs. 8.8 days), and hospital charges ( $\$ 19,686$ vs. $\$ 30,662$ ) significantly increased during S2 versus S1 (-Fig. 1). No differences in morbidity were observed in premature infants who were 3 to 6 and 6 to 12 months between seasons, and one infant died in each season. These results were further confirmed during the subsequent 2015 to 2016 season $(S 3)^{10}$ (-Fig. 1) which emphasizes the burden of RSV disease in this vulnerable population.

A large observational cohort study (SENTINEL 1) conducted during the 2014 to 2015 season in 702 infants born at 29 to 35 wGA who did not receive RSV immunoprophylaxis also showed that infants of lower gestational age and younger chronologic age were at high risk of RSV-associated hospitalizations, PICU admission, and need for mechanical ventilation. $^{11}$ 
Table 1 Summary of changes in the AAP guidelines on RSV immunoprophylaxis

\begin{tabular}{|l|l|l|}
\hline & $\mathbf{2 0 1 2}^{\mathrm{a}}$ & $\mathbf{2 0 1 4 ^ { \mathrm { b } }}$ \\
\hline$<29 \mathrm{wGA}$ & Age $<12$ months & Age $<12$ months \\
\hline $29-31 \mathrm{wGA}$ & Age $<6$ months & Not recommended \\
\hline $32-34 \mathrm{wGA}$ & $\begin{array}{l}\text { Age }<3 \text { months if } \geq 1 \text { risk factor } \\
\text { (3 doses) }\end{array}$ & Not recommended \\
\hline CLD & $\begin{array}{l}\text { Any GA and age }<24 \text { months of } \\
\text { CLD therapy in past } 6 \text { months }\end{array}$ & $\begin{array}{l}\bullet<32 \text { wGA and age }<12 \text { months }^{\text {d }} \\
\bullet<32 \text { wGA and age }<24 \text { months } \\
\text { df CLD therapy in past } 6 \text { months }\end{array}$ \\
\hline CHD & $\begin{array}{l}\text { Age }<24 \text { months with cyanotic } \\
\text { or acyanotic CHD }\end{array}$ & $\begin{array}{l}\text { Age }<12 \text { months with acyanotic CHD (recommended) or } \\
\text { cyanotic with CHF (may be considered) }\end{array}$ \\
\hline
\end{tabular}

Abbreviations: AAP, American Academy of Pediatrics; $\mathrm{CHD}$, congenital heart disease; $\mathrm{CHF}$, congestive heart failure; $\mathrm{CLD}_{\text {, chronic lung disease; } \mathrm{FiO}}$, fraction of inspired oxygen; wGA, weeks' gestational age.

${ }^{a} A A P$, RSV. In: Pickering LK, et al., eds. Red Book: 2012 Report of the Committee on Infectious Diseases. 29th ed. Elk Grove Village, IL, USA. 2. AAP Committee on Infectious Diseases; AAP Bronchiolitis Guidelines Committee. Pediatrics. 2014; 2:415-420

${ }^{b}$ American Academy of Pediatrics Committee on Infectious D, American Academy of Pediatrics Bronchiolitis Guidelines C. Updated guidance for palivizumab prophylaxis among infants and young children at increased risk of hospitalization for respiratory syncytial virus infection. Pediatrics. 2014; 134:415-20.

'Risk factors: daycare attendance and $\geq 1$ sibling aged $<5$ years.

${ }^{\mathrm{d}} \mathrm{CLD}$ defined as $\mathrm{FiO}_{2}>21 \%$ for $\geq 28$ days after birth.

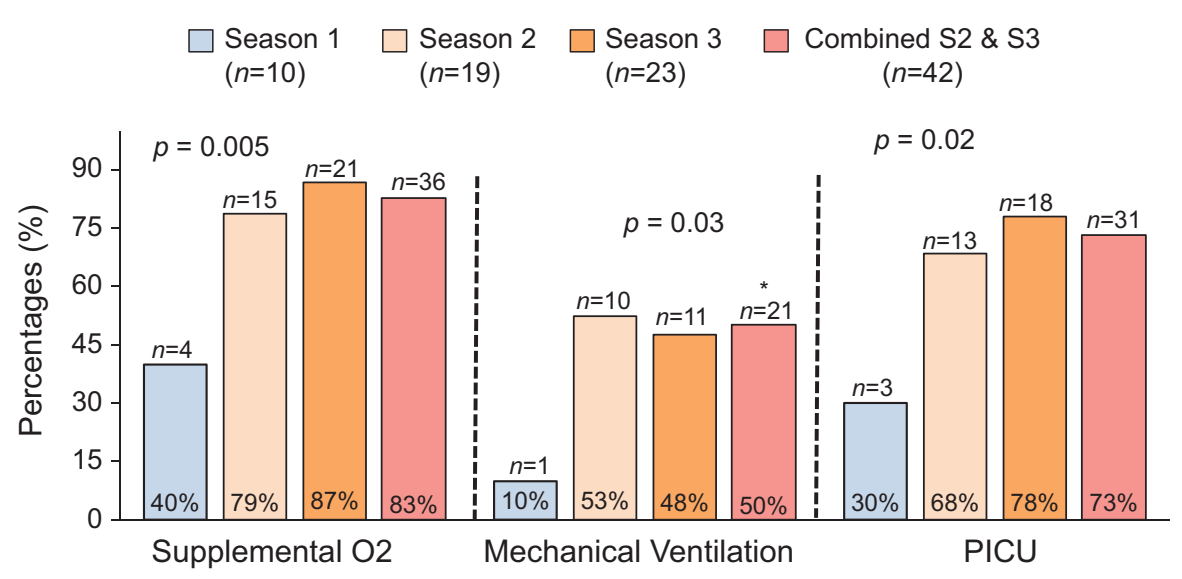

Fig. 1 Clinical outcomes in hospitalized infants $<3$ months of age born at 29 to 34 weeks of gestational age (wGA) during the season before the 2014 American Academy of Pediatrics (AAP) guidance change (S1) and two seasons after (S2, S3). Proportion of premature infants who received supplemental oxygen, mechanical ventilation, or pediatric intensive care unit (PICU) care during the 2013 to 2014 respiratory season (S1; before the 2014 AAP guidance change, light blue) versus the 2014 to 2015 (S2; fist season post-2014 AAP guidance change; light orange) and 2015 to 2016 (S3; second season post-2014 AAP guidance change; dark orange). Bars in pink represent the aggregate of the two seasons after the 2014 AAP guidance update. Y axis represents percentages and the number of patients is indicated above each column. The total number of premature infants $<3$ months of age hospitalized with respiratory syncytial virus lower respiratory tract infections is depicted underneath each season. $p$ values indicate significant differences between $\mathrm{S} 1$ and $\mathrm{S} 2 / \mathrm{S} 3$ by Kruskal-Wallis test.

Two recent large retrospective studies using commercial and Medicaid insurance databases compared RSV hospitalization rates in premature infants before and after the 2014 AAP guidance change, and included healthy full-term infants as a reference. ${ }^{12,13}$ Authors showed again that RSV-associated hospitalizations in infants born at 29 to 34 wGA and $<3$ months of age increased up to 2.7 -fold after the guidance change. They also showed that compared with fullterm infants, premature infants had up to seven times higher risk for RSV-associated hospitalizations. Together these studies suggest that the recommendations to narrow the indications for RSV immunoprophylaxis in high-risk preterm infants may have to be reconsidered. Whether the 2014 guidance update has also impacted infants with CLD or CHD needs to be further evaluated.

\section{Immunoprophylaxis with Other RSV Monoclonal Antibodies}

Other mAb for the prevention of RSV infection have been evaluated and/or are currently in clinical development. ${ }^{14}$ These include:

- Suptavumab (REGN-2222; Regeneron Pharmaceuticals Inc.) is a fully human monoclonal immunoglobulin G1 $\left(\operatorname{IgG}_{1}\right)$ antibody that targeted a well-conserved epitope of the preF protein (site $\mathrm{V}$ ). In vitro, suptavumab was almost 40 -fold more potent than palivizumab at inhibiting RSV fusion to respiratory epithelial cells and reducing lung and nasal RSV viral loads. ${ }^{15} \mathrm{~A}$ Phase III randomized, double-blind, placebo-controlled clinical trial conducted 
in premature infants born at $\leq 35$ weeks' gestation who were $\leq 6$ months of age at enrollment, and did not receive palivizumab, did not meet the primary end point (prevention of serious RSV LRTI). During the trial, RSV type B was the predominant circulating strain which developed specific escape mutations that conferred resistance to this $\mathrm{mAb}$, which is no longer being evaluated for clinical use.

- Nirsevimab (MEDI-8897; AstraZeneca/Medimmune/ Sanofi-Pasteur) is a G1 kappa (IgG1K) that targets a unique antigen site (site $\emptyset$ ) present exclusively on the preF conformation of the RSV F protein. It was derived from the human mAb D25 that showed in vitro to be 100 -fold more potent than palivizumab. ${ }^{16}$ In addition, using YTE technology MEDI-8897's half-life was extended to at least 150 days. A Phase IIb clinical trial in healthy 29 to 34 wGA preterm infants was completed and met the primary end point: a reduction in the incidence of medically attended LRTI due to RSV. This product is now undergoing Phase II/ III clinical trials in palivizumab-eligible patients, and Phase III trials in late preterm and full-term infants. The long-term goal of MEDI-8897 is to provide passive immunization for the prevention of RSV LRTI to all infants (preterm and full term) entering their first RSV season, using a fixed, single intramuscular dose.

- MK-1654 (Merck\& Co. Inc.) is an extended half-life human $\mathrm{mAb}$ directed against antigenic site IV, preserved in the preF and postF conformations of RSV F. MK-1654 is in early development for the prevention of RSV disease in both term and preterm infants.

\section{Conclusion}

Results from several randomized clinical trials, as well as from retrospective and prospective observational studies, add to the body of literature showing that premature infants of younger gestational and chronological age remain at high risk of severe RSV LRTI. Despite the consistent evidence of its clinical efficacy, one of the greatest barriers to a consensus regarding palivizumab prophylaxis is its high cost. The protection afforded by immunoprophylaxis has fueled interest in vaccinating pregnant women, with the goal of protecting the young infant through passive transfer of maternal antibodies. In fact, several RSV maternal vaccines trials are currently in clinical development. Regardless, passive immunoprophylaxis will still be needed and will complement the maternal vaccination strategy, especially in premature infants, as the majority of $\operatorname{IgG}$ transfer occurs in the last trimester of gestation. The development of newer cost-effective and long-acting $\mathrm{mAb}$ represents a promising advance for the prevention of RSV by providing enhanced efficacy while potentially improving patient adherence since fewer doses will be required. Ultimately, it will also reduce costs. These novel mAb may represent an opportunity for potential application to other patient populations such as otherwise healthy fullterm infants who represent the largest group hospitalized with RSV infection.

\section{Conflict of Interest}

A.M. has received research grants from NIH and Janssen; fees for participation in advisory boards from Janssen, Roche; and fees for lectures from Abbvie. O.R. has received research grants from NIH, the Bill \& Melinda Gates Foundation and Janssen; fees for participation in advisory boards from Merck, Sanofi-Pasteur, and Medimmune; and fees for lectures from Pfizer. P.J.S. has received research grants from NIH, Merck, and MedImmune, Inc., fees for participation in advisory boards from SanofiPasteur and AstraZeneca/Medimmune.

\section{References}

1 Shi T, McAllister DA, O'Brien KL, et al; RSV Global Epidemiology Network. Global, regional, and national disease burden estimates of acute lower respiratory infections due to respiratory syncytial virus in young children in 2015: a systematic review and modelling study. Lancet 2017;390(10098):946-958

2 Stockman LJ, Curns AT, Anderson LJ, Fischer-Langley G. Respiratory syncytial virus-associated hospitalizations among infants and young children in the United States, 1997-2006. Pediatr Infect Dis J 2012;31(01):5-9

3 García CG, Bhore R, Soriano-Fallas A, et al. Risk factors in children hospitalized with RSV bronchiolitis versus non-RSV bronchiolitis. Pediatrics 2010;126(06):e1453-e1460

4 Mejias A, Dimo B, Suarez NM, et al. Whole blood gene expression profiles to assess pathogenesis and disease severity in infants with respiratory syncytial virus infection. PLoS Med 2013;10(11): e1001549

5 McLellan JS, Chen M, Leung S, et al. Structure of RSV fusion glycoprotein trimer bound to a prefusion-specific neutralizing antibody. Science 2013;340(6136):1113-1117

6 Palivizumab, a humanized respiratory syncytial virus monoclonal antibody, reduces hospitalization from respiratory syncytial virus infection in high-risk infants. Pediatrics 1998;102(03):531-537

7 Feltes TF, Cabalka AK, Meissner HC, et al; Cardiac Synagis Study Group. Palivizumab prophylaxis reduces hospitalization due to respiratory syncytial virus in young children with hemodynamically significant congenital heart disease. J Pediatr 2003;143(04): 532-540

8 Blanken MO, Rovers MM, Molenaar JM, et al; Dutch RSV Neonatal Network. Respiratory syncytial virus and recurrent wheeze in healthy preterm infants. N Engl J Med 2013;368(19):1791-1799

9 Rajah B, Sanchez PJ, Garcia-Maurino C, Leber A, Ramilo O, Mejias A. Impact of the updated guidance for palivizumab prophylaxis against respiratory syncytial virus infection: a single center experience. J Pediatr 2017;181:183-188

10 Wozniak PS, Sanchez PJ, Rajah B, et al. Impact of the Revised Guidelines for Respiratory Syncytial Virus Prophylaxis: Morbidity Persists After Two Seasons. Infectious Diseases Society of America 54th Annual Meeting ID Week; 2016; New Orleans, LA.; 2016

11 Anderson EJ, Krilov LR, DeVincenzo JP, et al. SENTINEL1: an observational study of respiratory syncytial virus hospitalizations among U.S. infants born at 29 to 35 weeks' gestational age not receiving immunoprophylaxis. Am J Perinatol 2017;34(01): 51-61

12 Kong AM, Krilov LR, Fergie J, et al. The 2014-2015 national impact of the 2014 American Academy of Pediatrics guidance for respiratory syncytial virus immunoprophylaxis on preterm infants born in the United States. Am J Perinatol 2018;35(02):192-200

13 Goldstein M, Krilov LR, Fergie J, et al. Respiratory syncytial virus hospitalizations among U.S. preterm infants compared with term infants before and after the 2014 American Academy of Pediatrics guidance on immunoprophylaxis: 2012-2016. Am J Perinatol 2018;35(14):1433-1442 
14 Mejias A, Garcia-Maurino C, Rodriguez-Fernandez R, Peeples ME, Ramilo O. Development and clinical applications of novel antibodies for prevention and treatment of respiratory syncytial virus infection. Vaccine 2017;35(03):496-502

15 Simoes E. Phase 3, Randomized, Double-blind, Placebo-controlled Trial Evaluating the Efficacy and Safety of Suptavumab, for the Prevention of Medically attended RSV Infection in Preterm
Infants. 2018 Respiratory Syncytial Virus Symposium; 2018; Ashville, NC.; 2018

16 Domachowske JB, Khan AA, Esser MT, et al. Safety, tolerability and pharmacokinetics of MEDI8897, an extended half-life single-dose respiratory syncytial virus prefusion f-targeting monoclonal antibody administered as a single dose to healthy preterm infants. Pediatr Infect Dis J 2018;37(09):886-892 